\title{
Modelling and optimization of micro optofluidic lenses
}

\author{
Chaolong Song ${ }^{a}$, Nam-Trung Nguyen *a ${ }^{a}$,Say-Hwa Tan ${ }^{a}$, and Anand Krishna Asundi ${ }^{a}$ \\ Receipt/Acceptance Data [DO NOT ALTER/DELETE THIS TEXT] \\ Publication data [DO NOT ALTER/DELETE THIS TEXT] \\ ${ }_{5}$ DOI: 10.1039/b000000x [DO NOT ALTER/DELETE THIS TEXT]
}

This paper reports the modelling and experimental results of a liquid-core liquid-cladding optofluidic lens. The lens is based on three laminar streams in a circular chamber. The stream lines and the curvature of the interface can be predicted accurately using the theory of two-dimensional dipole flow in a circularly bounded domain. The model establishes basic relations between the flow rate ratio of the core/cladding 10 streams and the radius of curvature and consequently the focal length of the lens. Compared to a rectangular chamber, this new circular design allows the formation of liquid-core liquid-cladding lens with perfect curvatures. The circular design allows tuning a perfect curvature ranging from the chamber radius itself to infinity. The test device with a circular lens chamber with 1-mm diameter and $50-\mu \mathrm{m}$ height was fabricated in PDMS. The lens shape as well as the stream lines were characterized using 15 fluorescent dye and tracing particles. Experimental results agree well with the analytical results predicted by the model.

Keywords: micro optofluidics; liquid lens; liquid-core liquid-cladding lens; hydrodynamic sspreading; dipole flow

\section{Introduction}

20 Micro lenses with tuneable focal length can find a wide range of applications in lab-on-a-chip (LOC) systems. The integration of micro lenses into microfluidic system can improve the portability and make the system cost-efficient. Camou et al. used photolithography to fabricate PDMS 2D 25 optical lenses ${ }^{1}$, which is demonstrated to improve the performance of fluorescent spectroscopy detection. Wenger et al. explored the combination of a latex microsphere with low numerical aperture (NA) lens to enable the detection of of single molecules ${ }^{2}$. However their solid-based optical lenses

30 are not tuneable. The focal length is fixed after the fabrication of lens, and the smoothness of interface of the lens depends on the fabrication process. Liquid interfaces are atomically smooth and their curvature is maintained by the interfacial tension. The refractive indices of most liquid are in the range 35 of 1.3 to 1.6, which is comparable to glass and most polymeric materials. The smooth curved interfaces and the high refractive index make liquids ideal materials for designing lenses.

40 Liquid lenses with tuneable focal length have been reported in the past. The radius of curvature was controlled by pressure-driven deflection of a thin elastomeric membrane ${ }^{3,4}$ or by electrowetting. ${ }^{5,6}$ These lenses require an actuators for tuning the focal length. Thus, they are expensive to fabricate 45 and not compatible to most passive microfluidic systems. Recently, micro optofluidics has been emerging as an active

\footnotetext{
a School of Mechanical and Aerospace Engineering, Nanyang Technological University, 50 Nanyang Avenue, Singapore, 639798 E-mail:mntnguyen@ntu.edu.sg

$\dagger$ Electronic Supplementary Information (ESI) available:. See http://dx.doi.org/10.1039/b000000x/
}

research field, where liquid flows in microchannels are used to realize optical components. The small size of microchannels leads to a small Reynolds number and a stable 50 laminar flow. The smooth and relatively stable interfaces between different liquids are ideal for designing different optical components such as waveguides, lenses and mirrors. ${ }^{7}$ The simplest application of laminar liquid streams with mismatched refractive indices are liquid-core/liquid-cladding 55 waveguides. ${ }^{8}$ Hydrodynamic control of the liquid streams can be used to define the optical path. In the past, we have demonstrated the on-chip generation of a dye laser as well as the splitting and switching capability of the optical signal in a micro optofluidic device. ${ }^{9}$ The curved interface between two 60 immiscible liquids can be achieved with the contact angle at the liquid/liquid/solid interface ${ }^{5,6}$ or with the Dean flow in a curved channel. ${ }^{10}$ In the later case, a small radius of curvature and a short focal length can be achieved. Recently, Tang et al. reported a dynamically reconfigurable liquid-core liquid65 cladding lens using the curved interfaces in a rectangular chamber. ${ }^{11}$ This paper reported experimental results of the micro optofluidic lens formed by hydrodynamic spreading of a core stream, which has a higher refractive index than that of the side stream. The curvature of the lens is tuned by the flow 70 rate ratios of the three streams. The current design with a rectangular chamber suffers the problem of distortion of the lens shape due to recirculation flow at high flow rates. To the best of our knowledge, there is no theoretical works describing the interaction between the fluidic and the optical 75 aspects of this lens type.

In this paper, we propose a new design with a circular chamber to realize a perfect lens curvature. The paper focuses on the fluidic aspect of the lens design. First, a model 80 for the interface shape is established based on the theory of two- 


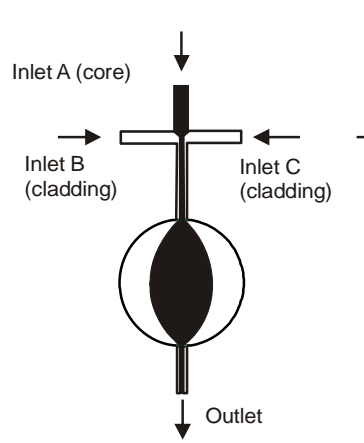

(a)

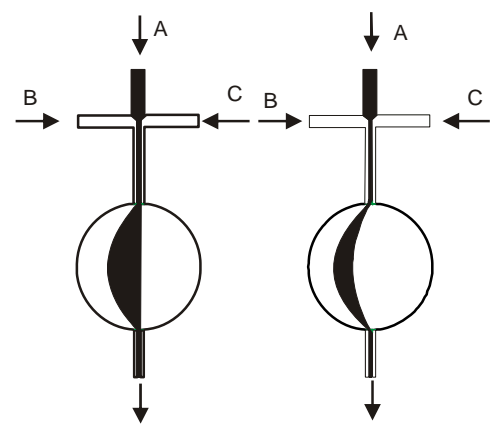

(b)

(c)
Fig. 1 The concept of the micro optofluidic lens with a circular lens chamber. The shapes and dimensions of the lens are tuneable by the flow rates of core and cladding streams: (a) double-convex lens; (b) planoconvex lens; (c) convergent-meniscus lens.

dimensional dipole flow in a circularly bounded domain. Next, the paper derives the basic relations between the flow rate ratio of the core/cladding streams and the radius of curvature and consequently the focal length of the lens. Although traditional ${ }_{85}$ precision engineering can fabricate optical lens with a pre-defined curvature of interface by using grinding and polishing, which is a mechanically-contact method, thus the optical smoothness is limited. Our model provides a method to configure optofluidic lens with a mathematically pre-defined curvature, whose interface 90 is automatically smooth. Experiments were carried out to verify the theory. The shape of the micro optofluidic lens was visualized using fluorescent dye and fluorescent particles.

\section{Model of liquid-core liquid cladding lens in a ${ }_{95}$ circular chamber}

\section{Dipole flow model for lens shape}

Figure 1 depicts the basic concept of a liquid-core liquidcladding optofluidic lens in a circular chamber. Our design based on liquid flow at low Reynold numbers, and thus 100 promises to form laminar streams in the lens chamber. The core stream sandwiched by two cladding streams enters the circular chamber and then expands to develop the lens-shape. Manipulating the flow rates of the three streams allows tuning the curvature of the interface of the lens, and consequently 105 changes the focal length. In our design, the width of the channel is much smaller than the dimension of the chamber. So the entrance and exit of the circular chamber act approximately as a source and sink, respectively. Therefore, the flow field in the circular chamber can be approximately 110 described by a source-sink pair model bounded in a circular domain. Based on this model, we calculate the curvature of the interface between core and cladding streams, and consequently the focal length of the fluidic lens.

115 Koplik et al. ${ }^{12}$ discussed the trajectories of tracer particles in a two-dimensional dipole flow field in a circularly bounded domain. In this source-sink pair model, a source of strength $Q$ is placed at $(-a, 0)$ in the Cartesian coordinates, and a corresponding sink of strength $-Q$ located at $(a, 0)$. The flow 120 field is determined by two parameters: the distance $2 a$ between the source and sink, and their strength $\mathrm{Q}$. This field can be described by the complex potential:

$$
W(z)=\phi+i \psi=\frac{Q}{2 \pi}\left(\log (z+a)-\log (z-a)+\log \left(R^{2}+a z\right)-\log \left(R^{2}-a z\right)\right)
$$

125 Where $x$ and $y$ are the coordinate in the two dimensional Cartesian system, $R$ is the radius of the circular domain and $z=x+i \cdot y$ with $i$ as the imaginary unit. The real part $\phi$ and imaginary part $\psi$ of complex potential are the velocity potential and stream function respectively. The streamlines 130 are equipotential lines of the function $\psi$.

In the case of the lenses depicted in Fig. 1, the source and sink can be assumed to be located at the circumference of the circular domain $(a=R)$. The complex potential can be written 135 as:

$$
W(z)=\frac{Q}{2 \pi}\left(\log (z+R)-\log (z-R)+\log \left(R^{2}+R z\right)-\log \left(R^{2}-R z\right)\right)
$$

The corresponding streamlines are shown in Fig. 2. For the mathematical simplicity, only the positive half of the $y$-axis $(y>0)$ is taken into consideration. In this region, the stream 140 function can be simplified into:

$\psi=\frac{Q}{2 \pi}\left(2 \tan ^{-1} \frac{y}{R+x}+2 \tan ^{-1} \frac{y}{R-x}-\pi\right)$

The streamlines are a cluster of curves $\psi=C_{i}(i=1,2,3 \ldots)$. Thus the coordinates $(x, y)$ at each streamline should satisfy the equation:

${ }^{145} \tan ^{-1} \frac{y}{R+x}+\tan ^{-1} \frac{y}{R-x}=\left(C_{i}+\pi\right) / 2$

For each specific $C_{i}$, equation (4) defines a curve representing a streamline. The two terms at the left hand side of the equation (4) are the angles $\alpha$ and $\beta$ depicted in Fig. 3(a). Since the sum of the angles $\alpha$ and $\beta$ is a constant for each 150 specific streamline, thus the angle $\angle M P N=\pi-(\alpha+\beta)$ is also a constant. According to the law of sines, the point $\mathrm{P}$ is located at a circle with a radius equal to $r=R / \sin (\alpha+\beta)$. Therefore, the track of point P satisfying equation (4) should be an arc of radius $R / \sin (\alpha+\beta)$. Thus, each streamline in Fig. 1552 is an arc with a radius $r$ :

$r=R / \sin (\alpha+\beta)$

$=R /\left(\frac{y}{\sqrt{(R+x)^{2}+y^{2}}} \frac{R-x}{\sqrt{(R-x)^{2}+y^{2}}}+\frac{R+x}{\sqrt{(R+x)^{2}+y^{2}}} \frac{y}{\sqrt{(R-x)^{2}+y^{2}}}\right)$

(5)

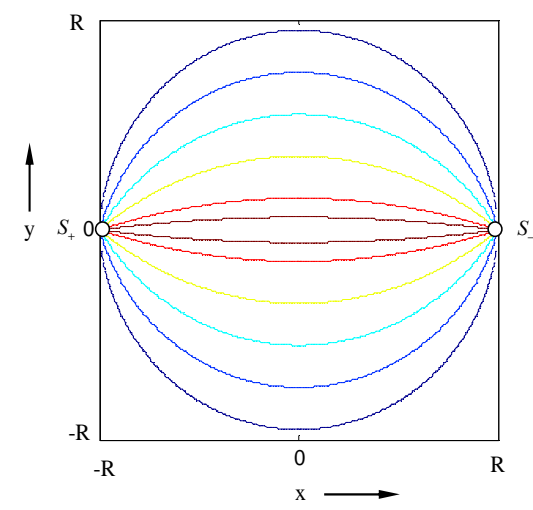

Fig. 2 Streamlines of source-sink pair in a circularly bounded domain with source and sink located at the circumference. 


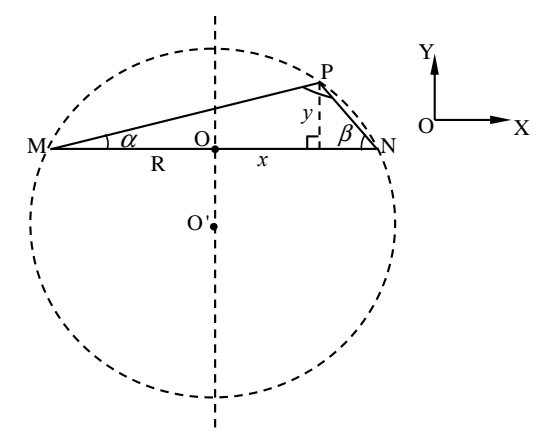

(a)

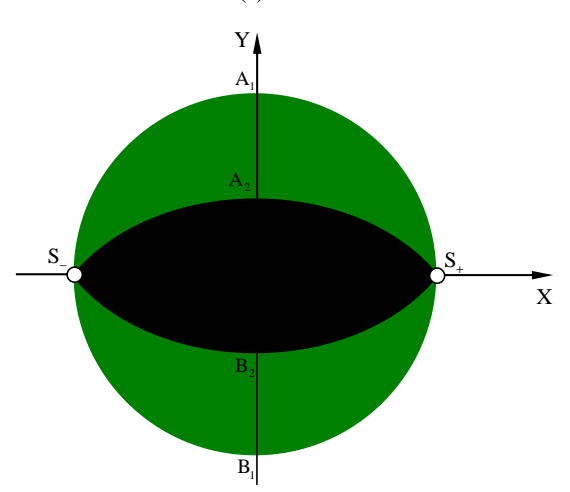

(b)

Fig. 3 (a) The coordinate of point $P$ satisfies the equation (4) and the trajectory of point $\mathrm{P}$ is an arc with a radius $r=R / \sin (\alpha+\beta)$; (b) Interface positions of a lens.

Because of symmetry, the streamlines in the other semiplane $(y<0)$ should also comply with the conclusion above. ${ }_{160}$ For streams with different viscosities, the interface position at $\mathrm{x}=0$ can be derived based on the theory of hydrodynamic focusing reported previously. ${ }^{9}$ With a given interface position, the radius of curvature can be determined using equation (5). A liquid interface with well-defined radius of curvature and a 165 mismatch in refractive indices can serve as an optofluidic lens. According to the above model, the curvature of the interface is tuneable by controlling the flow rate ratio of the streams. Following, the relationship between the flow rate ratio and the curvature of the interface are derived. For simplicity, the 170 following are derived for core liquids and cladding liquids with the same viscosity.

According to the theory of potential flow, the first derivative of the complex potential with respect to $z$ 175 represents the velocity field $\vec{V}=(u, v)$. The real and imaginary parts of this first derivative represent the velocity components in $x$-axis and $y$-axis, respectively. Differentiating $W$ in equation (2) with respect to $z$ results in the velocity components:

$\left\{\begin{array}{l}u=\frac{2(x+R)}{(x+R)^{2}+y^{2}}-\frac{2(x-R)}{(x-R)^{2}+y^{2}} \\ v=\frac{2 y}{(x+R)^{2}+y^{2}}-\frac{2 y}{(x-R)^{2}+y^{2}}\end{array}\right.$

At the centre cross-section of the chamber $(x=0)$ the velocity components are. $\left\{\begin{array}{l}u=\frac{4 R}{R^{2}+y^{2}} \\ v=0\end{array}\right.$

The curvatures of the two interfaces determined the 185 positions of $A_{2}$ and $B_{2}$ (Fig. 3(b)), and therefore decide the flow rates for core and cladding flows:

$$
\left\{\begin{array}{l}
\phi_{\text {cladding_C }}=\int_{A_{2}}^{A_{1}} u \cdot d y=\int_{A_{2}}^{A_{1}} \frac{4 R}{R^{2}+y^{2}} \cdot d y=4\left[\tan ^{-1}\left(y_{A_{1}} / R\right)-\tan ^{-1}\left(y_{A_{2}} / R\right)\right] \\
\phi_{\text {core }}=\int_{B_{2}}^{A_{2}} u \cdot d y=\int_{B_{2}}^{A_{2}} \frac{4 R}{R^{2}+y^{2}} \cdot d y=4\left[\tan ^{-1}\left(y_{A_{2}} / R\right)-\tan ^{-1}\left(y_{B_{2}} / R\right)\right] \\
\phi_{\text {cladding_B }}=\int_{B_{1}}^{B_{2}} u \cdot d y=\int_{B_{1}}^{B_{2}} \frac{4 R}{R^{2}+y^{2}} \cdot d y=4\left[\tan ^{-1}\left(y_{B_{2}} / R\right)-\tan ^{-1}\left(y_{B_{1}} / R\right)\right]
\end{array}\right.
$$

Simply by substituting $x=0$ into equation (5), the 190 relationship between the radius of interface and the position of intersection between interface and $y$-axis is retrieved by solving the equation

$$
y^{2}-2 r y+R^{2}=0
$$

or

${ }_{195}|y|=r-\sqrt{r^{2}-R^{2}}$

This relationship of radius of interface versus position of intersection is illustrated in Fig. 4. The position of the interface approaches the circumference of the chamber, the radius of interface approaches the radius of the chamber.

200 Suppose the radii for $S_{-} A_{2} S_{+}$and $S_{-} B_{2} S_{+}$are $r_{1}$ and $r_{2}$ respectively, according to equation (10), the positions of intersections between these arcs and y-axis are calculated as:

$\left\{\begin{array}{l}y_{A_{2}}=r_{1}-\sqrt{r_{1}^{2}-R^{2}} \\ y_{B_{2}}=\sqrt{r_{2}^{2}-R^{2}}-r_{2}\end{array}\right.$

Substituting (11) into (8) results in the fluxes of core and 205 cladding flows, which correspond to the stream flow rates:

$$
\left\{\begin{array}{l}
\phi_{\text {cladding_C }}=\int_{A_{2}}^{A_{1}} u \cdot d y=4\left[\pi / 4-\tan ^{-1}\left(\left(r_{1}-\sqrt{r_{1}^{2}-R^{2}}\right) / R\right)\right] \\
\phi_{\text {core }}=\int_{B_{2}}^{A_{2}} u \cdot d y=4\left[\tan ^{-1}\left(\left(r_{1}-\sqrt{r_{1}^{2}-R^{2}}\right) / R\right)-\tan ^{-1}\left(\left(\sqrt{r_{2}^{2}-R^{2}}-r_{2}\right) / R\right)\right] \\
\phi_{\text {cladding_B }}=\int_{B_{1}}^{B_{2}} u \cdot d y=4\left[\tan ^{-1}\left(\left(\sqrt{r_{2}^{2}-R^{2}}-r_{2}\right) / R\right)+\pi / 4\right]
\end{array}\right.
$$

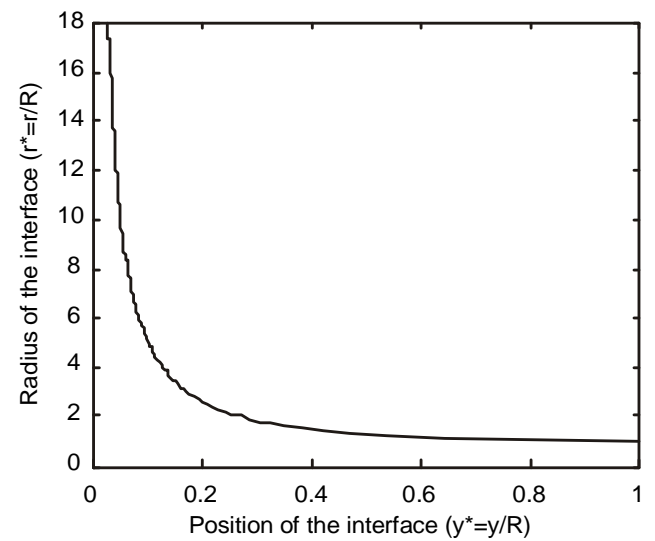

Fig. 4 Radius of interface versus the position of intersection between the corresponding interface and y-axis (both radius and position are normalized by the radius $R$ of the circular chamber). 


\section{Gaussian optics model}

210 In the case of the optofluidic lens, two optically smooth interfaces are formed by three streams of flow, and each interface has a constant curvature of radius. This structure can be simply and sufficiently described by a combination of two surfaces which are rotationally symmetric about the optical 215 axis as illustrated in Fig. 5. In this situation, the principal planes located across the surface vertices and their positions $l_{H}$ and $l_{H}^{\prime}$ are given as:

$\left\{\begin{array}{l}l_{H}=-f^{\prime} \frac{n-1}{n} \frac{d}{r_{2}} \\ l_{H}^{\prime}=-f^{\prime} \frac{n-1}{n} \frac{d}{r_{1}}\end{array}\right.$

where the $n$ is the refractive index of the lens, $d$ is the 220 distance between two surface vertices, $r_{1}$ and $r_{2}$ are the radii of the two interface, and $f^{\prime}$ is the focal length of the lens constructed by a combination of these two surfaces:

$f^{\prime}=\frac{n r_{1} r_{2}}{(n-1)\left[n\left(r_{2}-r_{1}\right)+(n-1) d\right]}$

Combining (13) and (14), the positions of focal points with 225 respect to front and rear surface vertices are respectively:

$\left\{\begin{array}{l}l_{F}=-f^{\prime}\left(1+\frac{n-1}{n} \frac{d}{r_{2}}\right) \\ l_{F}^{\prime}=-f^{\prime}\left(1-\frac{n-1}{n} \frac{d}{r_{1}}\right)\end{array}\right.$

Taking the cladding as the reference, the relative refractive indices of core and cladding streams are $n$ and 1, respectively.

230 Focal length is an important parameter to characterize a lens. At given refractive indices, the focal length is a function of the radii of the two interfaces. For mathematical simplicity, we confine the degrees of freedom to only one by assuming a case of symmetrical double-convex lens (Fig. 1 (a)) or fix one

235 radius of interface at a infinitely large value, which is actually the case of plano-convex lens, Fig. 1 (b). Then we discuss the flow rate ratios between core and cladding streams under a given focal length.

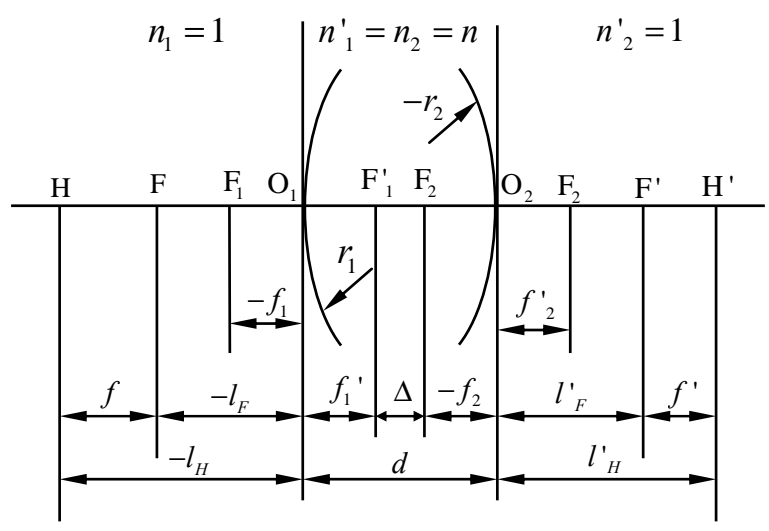

Fig. 5 A thick lens is constructed by a combination of two surfaces with given radii of curvature that are rotationally symmetrical about the optical axis.
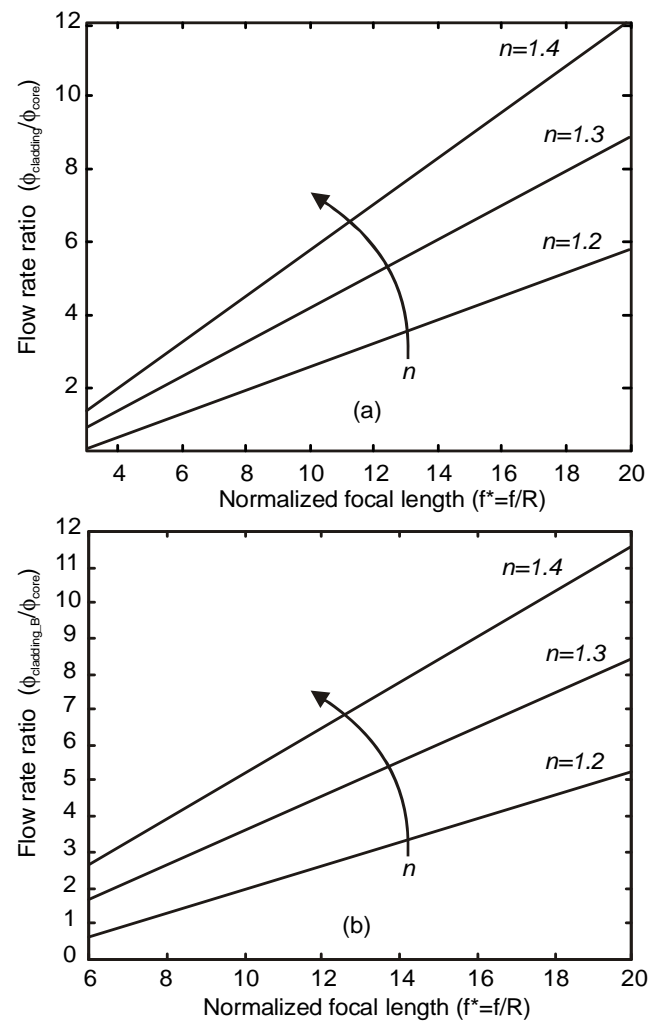

Fig. 6 Relationships between flow rate ratio and focal length (the dimension of focal length is normalized by the radius of circular chamber): (a) symmetrical double-convex lens (b plano-convex lens.

240 In the case of symmetrical double-convex lens (Fig. 1 (a)), the moduli of radii of two interfaces are both equal to $r$. The focal length is formulated as:

$f=\frac{-n r^{2}}{(n-1)[(n-1) d-2 n r]}$

Where $r$ is the radius of the interface of lens, $n$ is the 245 relative index of core stream, and $d$ is the distance between the vertices of two interfaces. In the case of $r>>d$, the focal length in equation (16) has an approximate expression:

$f=\frac{r}{2(n-1)}$

Under the assumption that the moduli of radii of two 250 interfaces are equal, the flow rate ratio between cladding and core streams according to (12) is:

$$
\frac{\phi_{\text {cladding }}}{\phi_{\text {core }}}=\frac{\pi}{8 \tan ^{-1}\left(\frac{r-\sqrt{r^{2}-R^{2}}}{R}\right)}-\frac{1}{2}
$$

Substituting relation (17) into (18), the relationship between the flow rate ratio and the focal length of the symmetrical 255 double-convex optofluidic lens is:

$$
\frac{\phi_{\text {cladding }}}{\phi_{\text {core }}}=\frac{\pi}{8 \tan ^{-1}\left(\frac{2(n-1) f-\sqrt{4(n-1)^{2} f^{2}-R^{2}}}{R}\right)}-\frac{1}{2}
$$

Figure 6 (a) depicts the relation between focal length and flow rate as well as refractive index. The focal length is almost linearly proportional to the flow ratio. At the same 260 refractive index, the higher the flow rate ratio between the cladding and the core, the longer is focal length. At the same 
flow rate ratio, the higher the refractive index the shorter is the focal length.

265 In the case of plano-convex lens as depicted Fig. 1 (b), one radius of an interface is infinitely large $r_{2}=\infty$; while the other has a finite dimension $r_{1}=r$. Then the focal length can be simplified into:

$\lim _{r_{2} \rightarrow \infty} f=\frac{r}{n-1}$

270 Under this assumption, the flow rate ratio between cladding $\mathrm{B}$ and core streams $\mathrm{A}$ is as following equations (12):

$\frac{\phi_{\text {cladding_B }}}{\phi_{\text {core }}}=\frac{\pi-\tan ^{-1} \frac{(n-1) f-\sqrt{(n-1)^{2} f^{2}-R^{2}}}{R}}{4 \tan ^{-1} \frac{(n-1) f-\sqrt{(n-1)^{2} f^{2}-R^{2}}}{R}}$

It is obvious that the cladding flow from inlet $C$ takes up a half space of the circular chamber, which means the flow rate 275 of stream $C$ is simply the sum of the flow rates of core stream A and cladding stream B. Figure 6 (b) shows the relationship between focal length and flow rate ratio between cladding $B$ and core $\mathrm{A}$.

\section{Experiments}

280 The test devices were fabricated in polydimethylsiloxane (PDMS) using the soft lithography technique. The mask was printed on a transparency film with a resolution of $8000 \mathrm{dpi}$. The transparency mask was subsequently used for defining the negative mould of the lens in a $50-\mu \mathrm{m}$ thick SU-8 layer. 285 PDMS was mixed from the two components with a weight ratio of $10: 1$, and then poured into the SU-8 mould. PDMS cured in vacuum oven at $60^{\circ} \mathrm{C}$ and cools at room temperature for 24 hours. The PDMS was then peeled off from the master mould, the $0.5-\mathrm{mm}$ diameter access holes were punched. The 290 moulded part was subsequently bonded to another flat PDMS part after treating both surfaces with oxygen plasma. Needles with an inner diameter of $0.33 \mathrm{~mm}$ and an outer diameter $0.64 \mathrm{~mm}$ were press fit into the access holes and worked as fluidic interconnects. With this technology, a circular lens 295 chamber with a diameter of $1 \mathrm{~mm}$ and a height of $50 \mu \mathrm{m}$ was realized. The width of the inlet and exit channels is $50 \mu \mathrm{m}$.

In our experiment, cladding liquid is de-ionized (DI) water mixed with $\mathrm{w} / \mathrm{w}$ fluorescence dye (fluorescein disodium salt ${ }_{300} \mathrm{C}_{20} \mathrm{H}_{10} \mathrm{Na}_{2} \mathrm{O}_{5}$, Acid Yellow 73 or C.I. 45350) and 3- $\mu \mathrm{m}$ red fluorescent particles (Duke Scientific Co.) were used to visualize the cladding liquid and its stream lines. The separated fluorescent bands of the fluorescent particles $(540 / 610 \mathrm{~nm})$ and of the dye $(490 / 520 \mathrm{~nm})$ allows easy 305 imaging by switching the epi-fluorescent attachments on the microscope (Nikon EclipseTE 2000-S, Japan). DI water works as the core liquid. The liquids were kept in 5-ml glass syringes, which are driven by two syringe pumps (KDS230, KD Scientific Inc, USA) which allow any flow rate ratio 310 needed. A sensitive CCD camera (HiSense MKII) attached to the microscope was used to capture the fluorescent images.

\section{Results and Discussions}

First, flow visualization with fluorescent particles verified that the streamlines in the circular chamber have a perfect arc315 shape, which is of great importance for designing the optofluidic lens. As mentioned above, red fluorescent particles are diluted in the two cladding flows that maintain the same flow rates as that of core flow. A sufficiently long exposure time of $85 \mathrm{~ms}$ was chosen, within which a particle 320 can travel from the inlet to the outlet of the circular chamber. In this way, the particles will leave streak line on the image as illustrated in the inset of Fig. 7. Since the flow is laminar in our case, the streak lines of the particles correspond to the streamlines of the flow in the chamber. We extract a group of 325 positions for each of three paths showed in Fig. 7, and use an arc to fit each of them. The agreement between the extracted positions and fitting arcs confirm that the streamlines in the circular chamber have a perfect arc-shape, which can not be achieved with a rectangular chamber. ${ }^{11}$

330

To verify the theory for predicting the relationship between the flow rate ratio and the curvature of the interface, blue light was used to excite the fluorescent dye dissolved in the cladding flows. The core flow with no fluorescent dye appears 335 black on the image. The interface between the dark and bright region was extracted, its curvature was also evaluated under different flow rate ratios between core and cladding flows

For the case of a symmetric lens as illustrated in Fig. 8, 340 the flow rate of core stream was fixed at $0.6 \mathrm{~mL} / \mathrm{h}$, while the flow rates of two cladding streams vary equally from $0.1 \mathrm{~mL} / \mathrm{h}$ to $1.2 \mathrm{~mL} / \mathrm{h}$. The corresponding Reynold number ranges from 0.44 to 1.67 , and Peclet number estimated for the fluorescent dye ranges from 444 to 1667 . By this way, a 345 symmetrical biconvex lens was achieved, and the curvature of interface is tuned by varying the flow rates of cladding streams. When the flow rates of cladding streams decrease, the interface of this fluidic lens approaches the wall of the chamber while still remain as an arc-shape. In the case of the 350 lens reported by Whitesides' group, ${ }^{11}$ the paraxial region of the lens was not bent with an perfect arc-shape, but flat especially when the interface approaches the wall of their rectangular chamber. Therefore our circular design to form the fluidic lens is demonstrated here to have inherently robust 355 wide-range tuneability. This advantage allows the realization

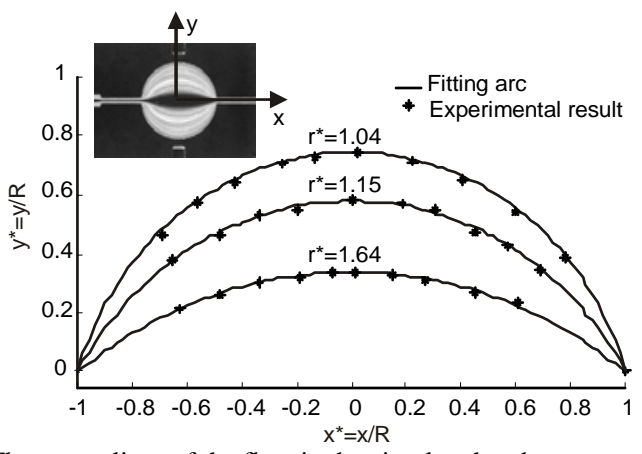

Fig. 7 The streamlines of the flow in the circular chamber are extracted by tracing of fluorescent particles. The asterisks in the figure represent the experimental results, the lines are the fittings arcs (all dimensions are normalized by the radius of the lens chamber). 
a smaller radius of interface, and thus a shorter focal length, which shows a potential to increase the level of integration of a lab-on-a-chip optical component.

The relationship between the flow rate ratio and the curvature of the interface can be analytically described by deriving Eq. (12):

$$
\frac{\phi_{\text {core }}}{\phi_{\text {cladding }}}=\frac{2 \tan ^{-1}\left(r / R-\sqrt{r^{2}-R^{2}} / R\right)}{\pi / 4-\tan ^{-1}\left(r / R-\sqrt{r^{2}-R^{2}} / R\right)}
$$

where $R$ is the radius of the circular chamber, and $r$ denotes 365 the radius of curvature of interface. Figure 8 shows the normalized curvature of the interface $(R / r)$ versus the flow rate ratio between the core and cladding stream. The results show that experimental data agrees well with the theoretical prediction. Thus, our analytical model of the optofluidic lens 370 can serve as a basic mathematic tool to design a fluidic lens if specific radii of interfaces are required.

To test the fluidic lens in the case of an asymmetric lens, we fixed the flow rates of the core and the upper cladding 375 streams equally at $0.6 \mathrm{~mL} / \mathrm{h}$ (insets in Fig. 9), and varied the flow rate of the lower cladding stream in a range from $0.15 \mathrm{~mL} / \mathrm{h}$ to $2.7 \mathrm{~mL} / \mathrm{h}$. The corresponding Reynolds number ranges from 0.75 to 2.17, and the Peclet number ranges from 750 to 2167. A plano-convex lens is achieved when the flow 380 rate of lower cladding stream is equal the sum of the flow rates of the core and the upper cladding stream or a flow rate ratio of 2 . When the flow rate ratio decreases, a biconvex lens is formed. If the flow rate ratio is larger than 2 , a meniscus lens can be constructed. With this test, different kinds of 385 lenses were demonstrated in our circular chamber design.

The curvatures of lower and upper interfaces can also be mathematically defined by flow rate ratio derived from Eq.

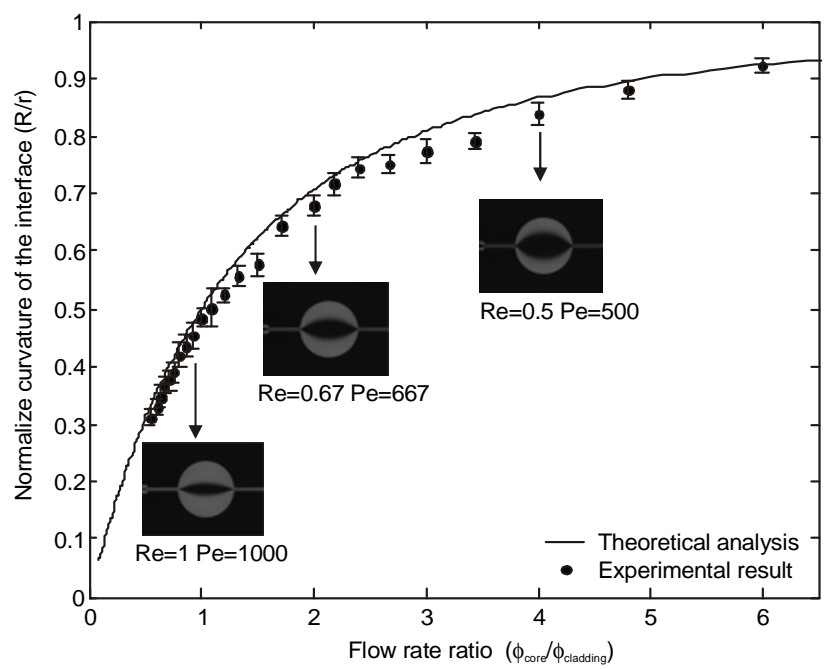

Fig. 8 Symmetrical case. The flow rates of two cladding streams are kept the same. The flow rate of core stream varies to achieve symmetrical biconvex lenses with different interface curvatures. The insets illustrate the fluorescence images of the lenses. The dimensions are normalized by the radius of circular chamber. The flow rate of core stream was kept at $0.6 \mathrm{~mL} / \mathrm{h}$, and the flow rate of cladding stream varies from $0.1 \mathrm{~mL} / \mathrm{h}$ to $1.2 \mathrm{~mL} / \mathrm{h}$. The corresponding Reynolds number ranges from 0.44 to 1.67 , and Peclet number ranges from 444 to 1667.

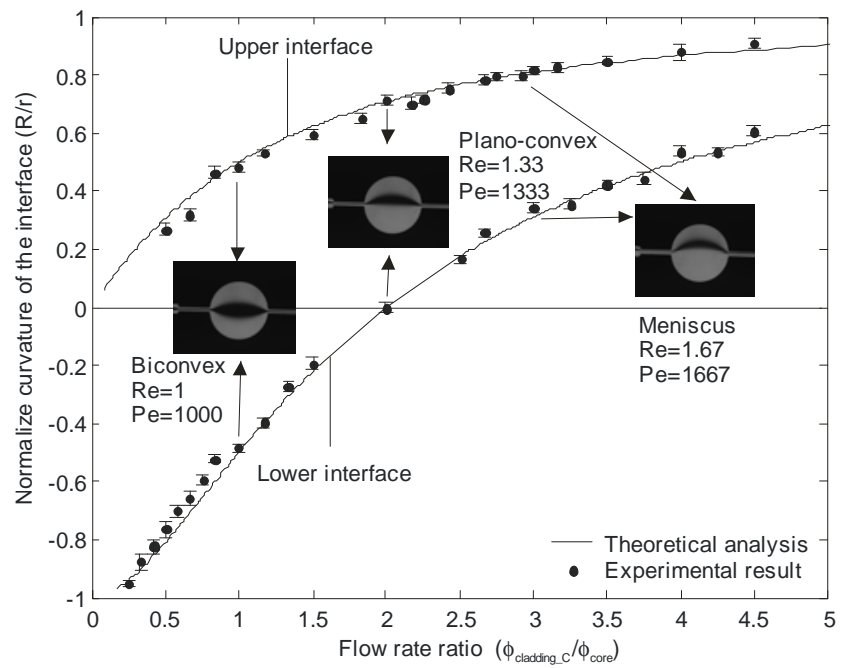

Fig. 9 Asymmetrical case. The flow rates of upper cladding and core streams are kept the same, and the flow rate of lower cladding stream varies to achieve biconvex, plano-convex, and meniscus lenses. The insets illustrate the fluorescence images of the lenses. The dimensions are normalized by the radius of the circular chamber. The flow rates of upper cladding and core streams are fixed equally at $0.6 \mathrm{~mL} / \mathrm{h}$, and the flow rate of lower cladding varies from $0.15 \mathrm{~mL} / \mathrm{h}$ to $2.7 \mathrm{~mL}$.h. The corresponding Reynold number ranges from 0.75 to 2.17 , and Peclet number ranges from 750 to 2167.

(12) assuming equal flow rates of the core stream and the 390 upper stream:

$$
\begin{aligned}
& \frac{\phi_{\text {lower_cladding }}}{\phi_{\text {core }}}=\frac{2 \tan ^{-1}\left(r_{1} / R-\sqrt{r_{1}^{2}-R^{2}} / R\right)}{\pi / 4-\tan ^{-1}\left(r_{1} / R-\sqrt{r_{1}^{2}-R^{2}} / R\right)} \\
& =\frac{\tan ^{-1}\left(r_{2} / R \pm \sqrt{r_{2}^{2}-R^{2}} / R\right)+\pi / 4}{\pi / 8-\tan ^{-1}\left(r_{2} / R \pm \sqrt{r_{2}^{2}-R^{2}} / R\right) / 2}
\end{aligned}
$$

where $r_{1}$ and $r_{2}$ are the radii of upper and lower interfaces, respectively. The solid lines in Fig. 9 depict the predicted radii of the two claddings versus the flow rate ratio between 395 lower cladding and core. We define the sign of curvature to be positive if the centre of curvature lies at the lower side of channel. The experimental results agree well with the theoretical analysis.

400 The device was also tested at higher flow rates, whose Reynold numbers range from 2 to 9 . When the Reynold number is relatively small, the flow can be fully developed in the circular chamber and follows stream lines predicted with the dipole theory. However with an increasing Reynold 405 number, the inertia causes flow separation at the entrance of the chamber. The flow passes through the chamber without following the streamlines as in low flow rate case. This phenomenon causes the lens to be shifted along the flow direction and the shape of the lens is distorted (Fig. 10). 410 Therefore working on a low Reynold number properly smaller than 2 is necessary for the lens to be well developed.

\section{Conclusions}

415 We reported the theoretical and experimental results of a micro optofluidic lens based on liquid-core liquid-cladding concept. The lens chamber was designed as a circular shape to 

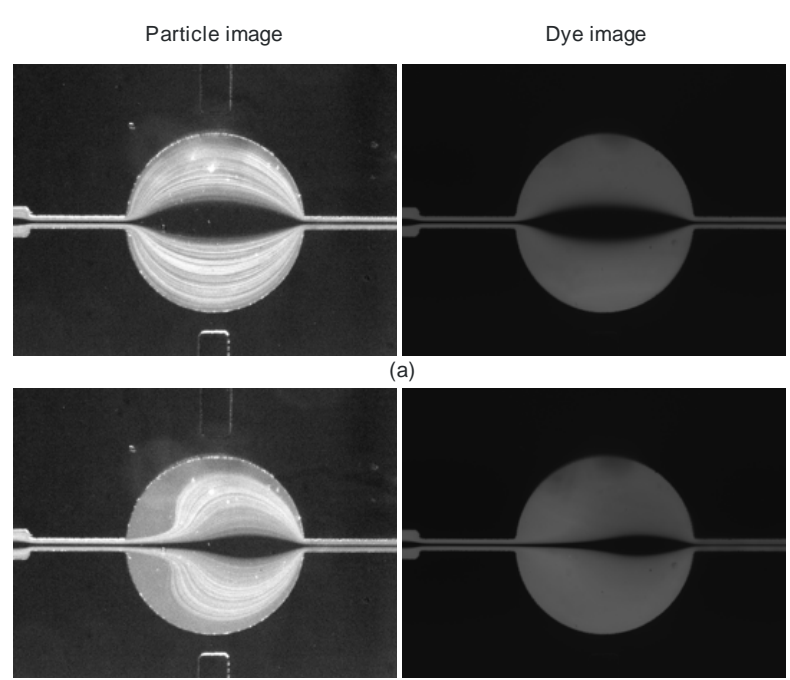

(b)

Fig. 10 Effect of flow separation on lens shape: (a) low flow rate 3.6 $\mathrm{mL} / \mathrm{h}(\mathrm{Re}=2)(\mathrm{b})$ high flow rate $16.2 \mathrm{~mL} / \mathrm{h}(\mathrm{Re}=9)$.

achieve a good curvature at the liquid interface. Experimental results validate the model describing the lens shape based on

420 the two-dimensional dipole flow theory. The model predicts the relations between the flow rate ratio between the core stream and the cladding stream and the radius of curvature and consequently the focal length of the lens. The design with a circular lens chamber allows the realization of an optofluidic 425 lens with a perfect arc shape. The test device was fabricated in PDMS. The lens chamber and the microchannels have a height of $50 \mu \mathrm{m}$. The streak lines of tracing fluorescent particles at low Reynolds numbers showed that the stream lines of the flow in the lens chamber follows a perfect arc. Experimental 430 results of the interface curvatures agree well with the analytical results predicted by the model.

\section{References}

1 S. Camou, H. Fujita, and T. Fujii, Lab Chip, 2003, 3, 40-45.

2 J. Wenger, D. Gerard, H. Aouani, and H. Rigneault, Anal. 435 Chem., 2008, 80, 6800-6804.

3 M. Agarwal, R. A. Gunasekaran, P. Coane and K. Varahmyan, J. Micromech. Microeng., 2004, 14, 1665-1673.

4 L. Pang, U. Levy, K. Campbell, A. Groisman and Y. Fainman, Optics Express, 2005, 13, 7928

4405 S. Kuiper and B.H.W. Hendriks, Applied Physics Letters, 2004, 85, 1128-1130.

6 C.C. Cheng and J.A. Yeh, Optics Express, 2007, 15, 82140.

7 D. Psaltis, S.R. Quake and C. Yang, Nature, 2006, 442, 381386.

4458 D. B. Wolfe, R.S. Conroy, P. Garstecki, B.T. Mayers, M.A. Fischbach, K.E. Paul, M. Prentiss and G.M. Whitesides, Proc. Natl Acad. Sci., 2005, 101, 12434-12438.

9 N.T. Nguyen, T. F. Kong, J. H. Goh and C. L. N. Low, J. Micromech. Microeng., 2007, 17, 2169-2174.

45010 X.L. Mao. J. R. Waldeisen, B. K. Juluri and T.J. Huang, Lab Chip, 2007, 7, 1303-1308.

11 S.K.Y. Tang, C.A. Stan and G. M. Whitesides, Lab Chip, 2008, 8, 395-401.

12 J. Koplik, S. Redner, and E. J. Hinch, Phys. Rev. E, 1994, 50, 455 4650-4671. 